\title{
A NOTE ON POLYNOMIAL HULLS
}

\author{
H. AL.EXANDER ${ }^{1}$
}

Abstract. Some properties of polynomially convex hulls of compact sets in $\boldsymbol{C}^{n}$ are deduced by simple topological arguments applied to known topological facts about polynomially convex sets.

Introduction. It is well known that the polynomial convexity of a compact set in $C^{n}$ entails certain topological restrictions (see below). Thus, for example, a 2 -sphere in $C^{2}$ can never be polynomially convex and, consequently, the problem of describing the huil presents itself. In the case of a differentiable 2-sphere in $C^{2}, E$. Bishop [1] has shown that the hull contains a family of analytic discs bounded by curves in the 2sphere; in particular, the hull has topological dimension at least 3 . The purpose of this note is to derive some properties of hulls by means of simple topological reasonings. We shall not produce analytic structure in the hull, but rather merely specify its extent. The first result generalizes the dimension increase known in the differentiabie case.

THEOREM 1. Let $X \subseteq C^{n}$ be compact and suppose $H^{k}(X, C) \neq 0$ for some $k \geqq n$. Then the topological dimension of $\hat{X} \backslash X$ is $\geqq k+1$. (Here $\dot{X}$ denotes the polynomially corvex hull.)

In the case of a manifold further information is obtained:

THEOREM 2. Let $M \subseteq C^{n}$ be a compact orientable topological $k$-manifold for $k \geqq n$. Then $(\hat{M} \backslash M)-\supseteq M$.

We note that this is not a local result as $M$ may well be locally polynomially convex near some point.

Let $B$ be the open unit (Euclidean) ball in $C^{n}$ ( $\left.n \geqq 2\right)$.

THEOREM 3. Let $X$ be a compact subset of $\partial B$. Then $X$ disconnects $\partial B$ if and only if $\hat{X}$ disconnects $B$.

Demonstrations. The basic fact which we shall need from analysis is due to A. Browder [2]:

(*) $H^{k}(K, C)=0$ for $k \geqq n$ if $K$ is a compact polynomially convex subset of $C^{n}$.

Received by the editors November $1,1970$.

AMS 1970 subject classifications. Primary 32E20.

Key words and phrases. Polynomially convex huli, several complex variables.

${ }^{1}$ This work was done under the sponsorship of NSF Grant No. GP-19067.

(c) American Mathematical Society 1972 
Proof of Theorem 1. We write the exact cohomology sequence for the pair $(\hat{X}, X)$ :

$$
H^{k}(\hat{X}, C) \rightarrow H^{k}(X, C) \rightarrow H_{*}^{k+1}(\hat{X} \backslash X, C) .
$$

Here $H_{*}$ denotes cohomology with compact supports (see [3, p. 190]). By $\left({ }^{*}\right)$ the first group is trivial and by assumption the second group is nontrivial. Hence $H_{*}^{k+1}(\hat{X} \backslash X, C) \neq 0$ and so $\operatorname{dim}(\hat{X} \backslash X) \geqq k+1$ (cf. [3, p. 236]).

Proof of Theorem 2. We argue by contradiction. That is, we set $A=(\hat{M} \backslash M)^{-}, Y=M \cap A$, and suppose that $Y$ is a proper subset of $M$. Consider the exact cohomology sequence of the pair $(\hat{M}, M)$ (we shall suppress the coefficient group $C$ everywhere below).

$$
H^{k}(\hat{M}) \rightarrow H^{k}(M) \rightarrow H^{k+1}(\hat{M}, M) \rightarrow H^{k+1}(\hat{M}) .
$$

By $\left({ }^{*}\right)$ the extremities are trivial. As $H^{k}(M) \neq 0$, we have $H^{k+1}(\hat{M}, M) \neq 0$. By excision $H^{k+1}(\hat{M}, M)=H^{k+1}(A, Y)$. Next consider the sequence for the pair $(A, Y)$ :

$$
H^{k}(Y) \rightarrow H^{k+1}(A, Y) \rightarrow H^{k+1}(A) \rightarrow H^{k+1}(Y) .
$$

It can be seen that $H^{k}(Z)=0$ for any proper closed subset of $M$ and so $H^{k}(Y)=0$; also $H^{k+1}(Y)=0$ as $\operatorname{dim} Y \leqq k$. Hence $H^{k+1}(A) \approx$ $H^{k+1}(A, Y) \neq 0$. Consider now the sequence for the pair $(\hat{M}, A)$ :

$$
H^{k+1}(\hat{M}) \rightarrow H^{k+1}(A) \rightarrow H^{k+2}(\hat{M}, A) \rightarrow H^{k+2}(\hat{M}) .
$$

By $\left(^{*}\right)$ the extremities are trivial and so $H^{k+2}(\hat{M}, A) \approx H^{k+1}(A) \neq 0$. By excision $0 \neq H^{k+2}(\hat{M}, A)=H^{k+2}(M, Y)$. Finally for the pair $(M, Y)$ :

$$
H^{k+1}(Y) \rightarrow H^{k+2}(M, Y) \rightarrow H^{k+2}(M) .
$$

The extremities are trivial and therefore so is $H^{k+2}(M, Y)$, a contradiction.

Q.E.D.

Proof of Theorem 3. By Alexander duality, $\tilde{H}_{0}(\partial B \backslash X)=H^{2 n-2}(X)$. Let $E^{2 n}$ be the cone on $\partial B$ and let $T=E^{2 n} \cup \hat{X}$. Consider the cohomology sequence for the pair $(T, \hat{X})$ :

$$
H^{2 n-2}(\hat{X}) \rightarrow H^{2 n-1}(T, \hat{X}) \rightarrow H^{2 n-1}(T) \rightarrow H^{2 n-1}(\hat{X}) .
$$

By $\left(^{*}\right)$, the extremities are trivial and so $H^{2 n-1}(T, \hat{X}) \approx H^{2 n-1}(T)$. By excision $H^{2 n-1}(T, \hat{X})=H^{2 n-1}\left(E^{2 n}, X\right)$. For the pair $\left(E^{2 n}, X\right)$ :

$$
H^{2 n-2}\left(E^{2 n}\right) \rightarrow H^{2 n-2}(X) \rightarrow H^{2 n-1}\left(E^{2 n}, X\right) \rightarrow H^{2 n-1}\left(E^{2 n}\right) .
$$

Again the extremities are trivial and so $H^{2 n-1}\left(E^{2 n}, X\right)=H^{2 n-2}(X)$. Hence $H^{2 n-1}(T)=H^{2 n-2}(X)$. By Alexander duality, $H^{2 n-1}(T)=\tilde{H}_{0}\left(\left(E^{2 n} \cup B\right) \backslash T\right)$. Since $\left(E^{2 n} \cup B\right) \backslash T=B \backslash \hat{X}$, we get $\tilde{H}_{0}(\partial B \backslash X)=\tilde{H}_{0}(B \backslash \hat{X})$. That is, $\partial B \backslash X$ and $B \backslash \hat{X}$ have the same number of components. Q.E.D. 
REMARK. As John Garnett has pointed out to me, the proof actually shows that $H_{i}(\partial B \backslash X)=H_{i}(B \backslash \hat{X})$ for $i \leqq n-2$.

\section{REFERENCES}

1. E. Bishop, Differentiable manifolds in complex Euclidean space, Duke Math. J. 32 (1965), 1-21. MR 34 \#369.

2. A. Browder, Cohomology of maximal ideal spaces, Bull. Amer. Math. Soc. 67 (1961), 515-516. MR 24 \#A440.

3. R. Godement, Topologie algébrique et théorie des faisceaux, Hermann, Paris, 1964.

Department of Mathematics, University of California, los Angeles, CaliFORNIA 90024

Department of Mathematics, University of Michigan, Ann Arbor, Michigan 48104 